\title{
Video Article \\ Microinjection of Medaka Embryos for use as a Model Genetic Organism
}

\author{
Sean R. Porazinski ${ }^{1}$, Huijia Wang ${ }^{1}$, Makoto Furutani-Seiki ${ }^{1}$ \\ ${ }^{1}$ Centre for Regenerative Medicine, Department of Biology and Biochemistry, University of Bath
}

Correspondence to: Makoto Furutani-Seiki at mfs22@bath.ac.uk

URL: https://www.jove.com/video/1937

DOI: doi:10.3791/1937

Keywords: Developmental Biology, Issue 46, medaka, zebrafish, evolution, mutant, vertebrate, genome function

Date Published: $12 / 22 / 2010$

Citation: Porazinski, S.R., Wang, H., Furutani-Seiki, M. Microinjection of Medaka Embryos for use as a Model Genetic Organism. J. Vis. Exp. (46), e1937, doi:10.3791/1937 (2010).

\section{Abstract}

In this video, we demonstrate the technique of microinjection into one-cell stage medaka embryos. Medaka is a small egg-laying freshwater fish that allows both genetic and embryological analyses and is one of the vertebrate model organisms in which genome-wide phenotype-driven mutant screens were carried out ${ }^{1}$, as in zebrafish and the mouse. Divergence of functional overlap of related genes between medaka and zebrafish allows identification of novel phenotypes that are unidentifiable in a single species ${ }^{2}$, thus medaka and zebrafish are complementary for genetic dissection of vertebrate genome functions.

To take advantage of medaka fish whose embryos are transparent and develop externally, microinjection is an essential technique to inject celltracers for labeling cells, mRNAs or anti-sense oligonucleotides for over-expressing and knocking-down genes of interest, and DNAs for making transgenic lines.

\section{Video Link}

The video component of this article can be found at https://www.jove.com/video/1937/

\section{Protocol}

\section{Mating and collecting eggs}

1. Female and male medaka can easily be distinguished by the size and shape of the anal and dorsal fins. The anal fins of the males are larger and parallelogram-shaped compared to that of the females. The female anal fin is smaller and triangle shaped. The male dorsal fin has a clearly visible deep notch between the last two rays.

2. Medaka lay up to 40 eggs every day and medaka females carry eggs clustered at their belly by attachment filaments for several hours before they are stripped off at plants in the tank.

3. Females can be caught in a net and held inside gently from both sides by one hand. Eggs can then be gently teased from the belly with the index finger of the other hand. The female can then be returned to the tank. Eggs should be transferred to a $6 \mathrm{~cm}$ Petri dish filled with embryo media by blunt forceps or a finger.

4. Medaka pairs rarely fight when they are kept in a small mating box. Therefore, they can be kept in one small tank with water flow so that they can be fed and eggs can be collected from the same pair every day for a couple of months.

5. It is important that female fish should not be kept without males, since ovulation occurs everyday in medaka females. Otherwise, females cannot deliver eggs and become unwell.

\section{Cleaning embryos}

1. Transfer unclustered eggs with a glass pipette to sandpaper (p2000 grit size, waterproof) placed in the lid of a $9 \mathrm{~cm}$ Petri dish. Remove excess medium but ensure a sufficient volume remains as to prevent drying of embryos.

2. Gently roll embryos on sandpaper using the forefinger, applying minimal pressure and keeping finger parallel to the surface of the sand paper for around 45-60 seconds to remove some of the outer surface hairs of the chorion. Do not roll more than 5-7 embryos at once this precaution will minimize the risk of crushing embryos beneath each other.

3. Transfer cleaned embryos to a fresh dish of embryo medium.

\section{Making agarose plates for holding embryos during microinjection}

Embryos are held in troughs during injection made with a plexiglass mould in 1.5\% agarose (Figure 1). The concentration of agarose is important to hold embryos properly. 
1. First, make a frame of agarose to hold the plexiglass mould. Pour $1.5 \%$ agarose in water to a $0.5 \mathrm{~cm}$ depth in a $9 \mathrm{~cm}$ Petri dish and wait until it completely solidifies. Cut out an area of the agarose just larger than the size of the mould.

2. Pour $1.5 \%$ agarose to fill the cut area and place the mould so that no bubbles are trapped and wait until agarose solidifies. This agarose plate can be stored in the refrigerator to speed up solidifaction.

3. Remove the mould to create troughs to hold the eggs. Add enough embryo medium to immerse the mould, cover and store at $4^{\circ} \mathrm{C}$ until needed.
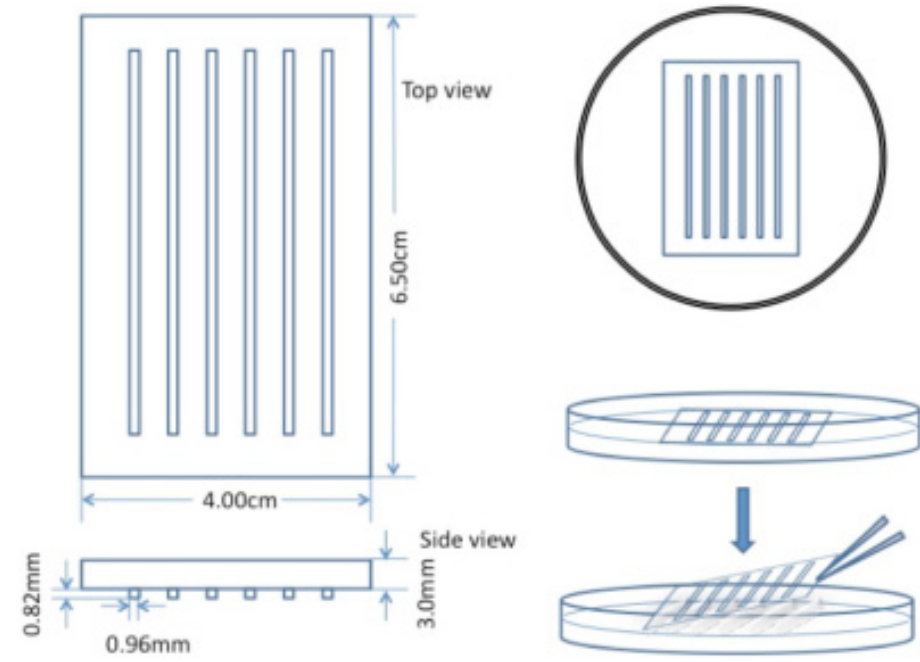

Figure 1. Schematic of custom-made mould used to prepare troughs in agarose plates for microinjection.

\section{Microinjection of medaka embryos}

In medaka, DNA, RNA, morpholino oligonucleotides and tracer dyes are microinjected through the chorion into the cytoplasm of the 1 to 64 -cell stage embryo rather than the yolk as in zebrafish. Since the cytoplasm is smaller and less visible in medaka, practice by injecting dyes such as rhodamine-dextran is recommended to develop this skill. Addition of phenol red as a tracer is also encouraged to allow confirmation of injections.

As the chorion surrounding medaka embryos is tough, a short, strong and wide injection needle is required (Figure 2). Prepare this strong wide injection needle from a filament-containing glass capillary.

1. Prepare injection solution with phenol red as a tracer and load it into the needle from the back with an Eppendorf microloader. Connect the needle to the air pump injector.

2. Eggs must be collected and unclustered as soon as possible after fertilization to inject at the one-cell stage. More embryos can be injected by storing them on ice (not more than 30 minutes) to stop cell division.

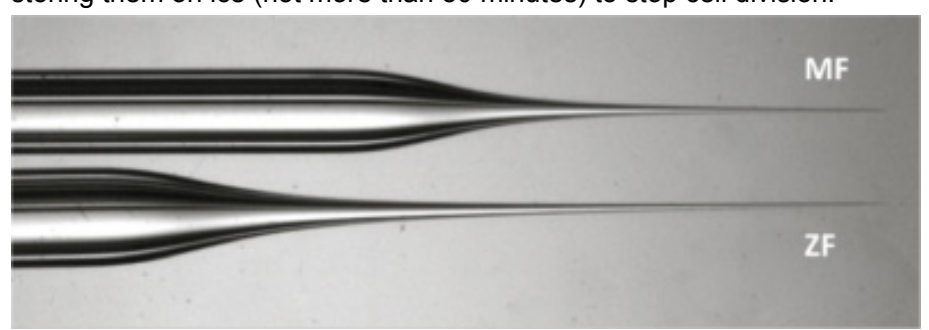

Figure 2. Comparison of medaka and zebrafish needles. Note the tip of the MF needle (top) is shorter and wider hence stronger for puncturing the tough chorion surrounding medaka embryos.

3. Transfer eggs into the agarose plate and align them in the troughs with forceps. Just before injection, five eggs should be rotated so the cell membrane of the one-cell cytoplasm can be hit by the needle perpendicularly. The cytoplasm of the one-cell can be found by looking for an area devoid of small oil droplets which is opposite to the side of the egg with densest oil droplets. The identified area can be confirmed to be the cytoplasm by viewing from the side.

4. Place the needle close to the eggs. Open the tip of the needle by gently touching the tip of the needle to the chorion or using microforceps (Figure 3). A small amount of injection substance should be seen to flow out.

5. Puncture through the chorion to place the tip of the needle inside of the chorion. Adjust the holding pressure of the injector to be relatively high, to ensure no reflux occurs due to the high pressure upon puncture through the tough chorion (settings of 80-100hPA for holding pressure and 500-700hPA for injection pressure).

6. Insert the tip of the needle into the cytoplasm by sharply poking its membrane.

Avoid inserting the tip of the needle into the yolk. Unlike zebrafish, material injected into the yolk will not be transferred into the cytoplasm. Injected liquids have a distinct boundary when injected into the yolk whereas the boundary is blurred when in the cytoplasm (Figure 4). Occasionally the needle may become blocked by agarose/debris. Forceps can be used to touch/rebreak the end of the needle and remove the blockage. If the needle breaks at any point during injection, bubbling will be seen in the embryo medium and the substance you are injecting will be lost. Injections should be carried out systematically from top to bottom along agarose channels and from left to right across the mould.

7. Injected embryos should be transferred to a clean dish containing embryo medium and developed at the appropriate temperature. 

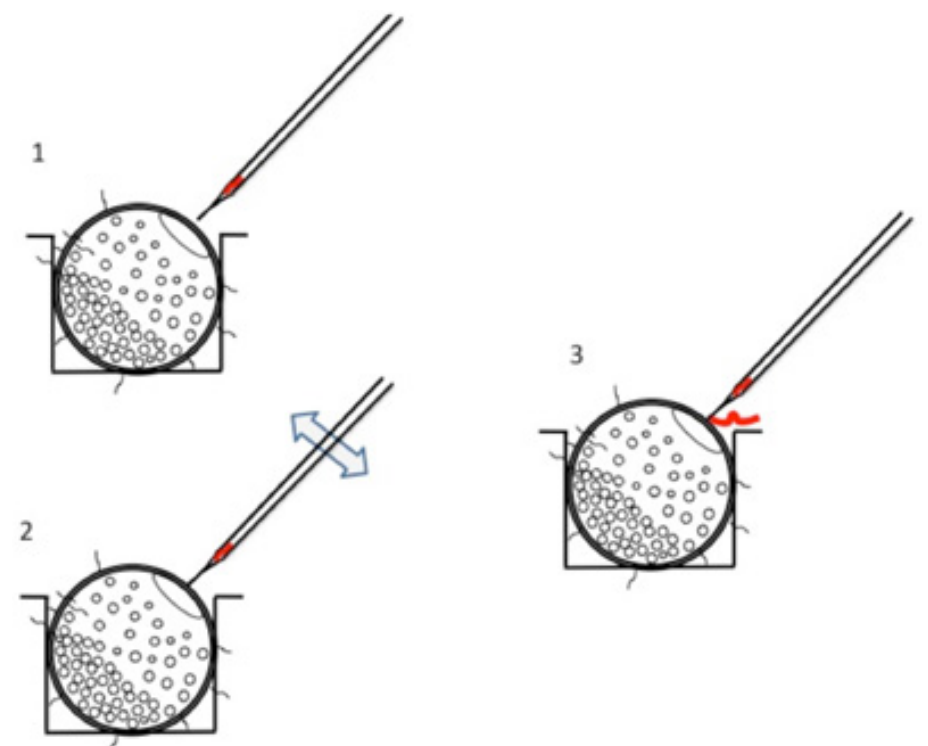

Figure 3. Breaking the needle tip prior to injection. 1: Move tip of the needle close to the chorion of embryo; 2: Touch the chorion and move needle backwards and forwards gently to break tip; 3 : When a small amount of injection substance flows out of the end of the needle this confirms breakage of the tip.

\section{Representative results}

\section{Correct injection Incorrect injection}
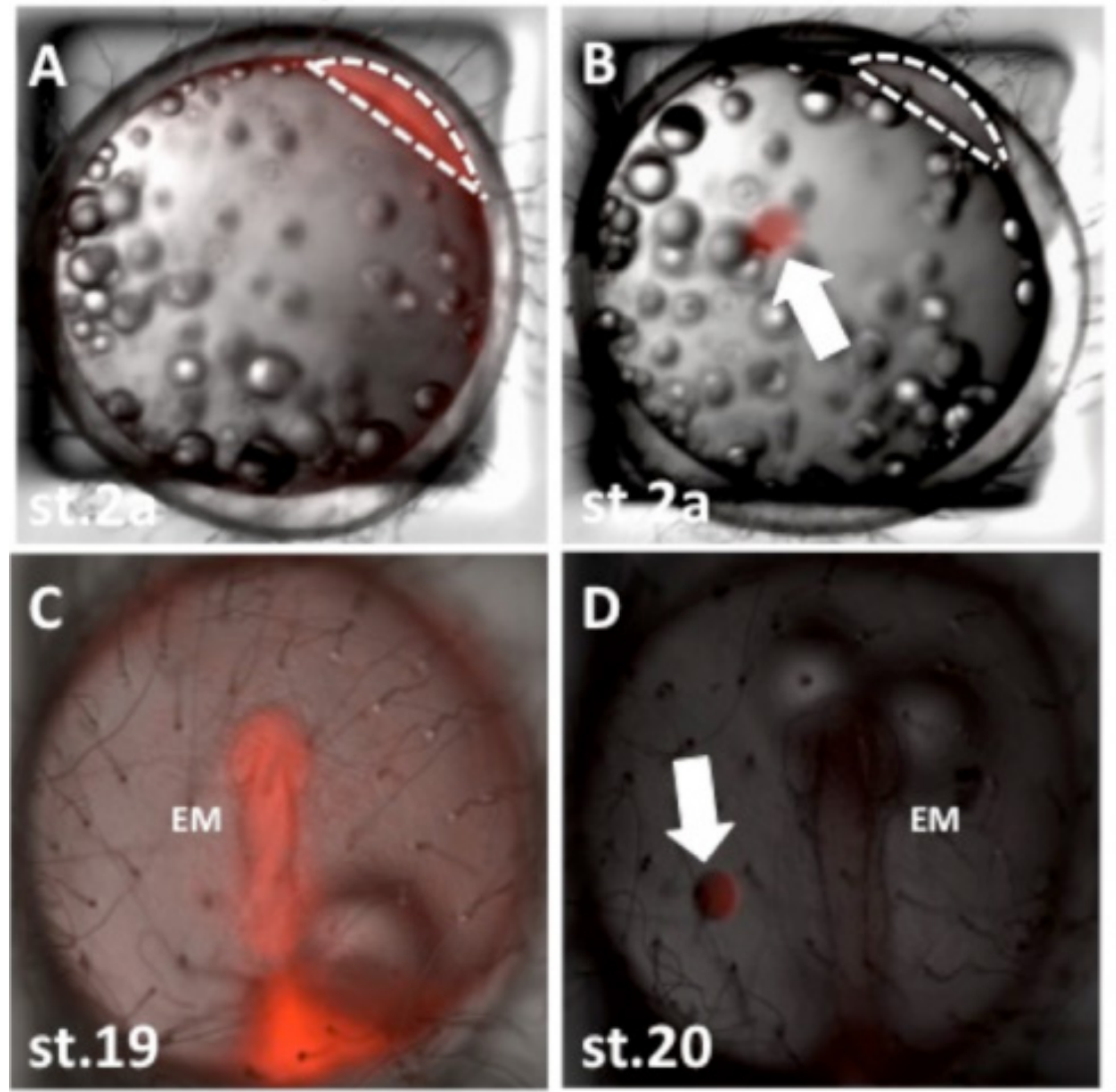

\section{st.19}

Figure 4. Representative images of injected medaka embryos. Panel A shows a representative image of correct injection of rhodamine-dextran into the cytoplasm of a one-cell stage medaka embryo. Panel B shows a representative image of incorrect injection of rhodamine-dextran into the yolk sac of a one-cell stage medaka embryo. Panels $C$ and $D$ show the embryos from panels $A$ and $B$ respectively at approximately 30 hours post-injection. EM: embryo, anterior is upwards in panels $C$ and $D$ and view is dorsal. Note the body of the embryo in panel $D$ is not red as the 
dye was incorrectly injected into the yolk sac. Arrows in B and D indicate rhodamine-dextran injected incorrectly into the yolk sac note the distinct circular boundary. Dotted lines in A and B indicate outline of cytoplasm of one-cell, view is lateral.

\section{Discussion}

In this video, we demonstrate a method for cell tracer, mRNA, DNA or anti-sense oligonucleotide injection into one-cell stage medaka embryos. This technique has allowed us to study various developmental processes in-vivo in real-time. This process and the subsequent detailed analysis it affords has the potential to significantly enhance our understanding of vertebrate organogenesis and the underlying cell biology. Such knowledge may be important for, and yield therapeutic applications in regenerative medicine.

\section{Acknowledgements}

This work was supported by a grant from the MRC.

\section{References}

1. Furutani-Seiki, M., Sasado, T., Morinaga, C., et al. A systematic genome-wide screen for mutations affecting organogenesis in Medaka, Oryzias latipes. Mech Dev 121, 647-58 (2004).

2. Furutani-Seiki, M., and Wittbrodt, J. Medaka and zebrafish, an evolutionary twin study. Mech. Dev. 121, 629-37 (2004). 